\title{
Ethanol extract of propolis protects macrophages from oxidized low density lipoprotein-induced apoptosis by inhibiting CD36 expression and endoplasmic reticulum stress-C/EBP homologous protein pathway
}

Hua Tian', Hong-Wei Sun², Jia-Jun Zhang ${ }^{3}$, Xiao-Wei Zhang ${ }^{3}$, Li Zhao ${ }^{1}$, Shou-Dong Guo ${ }^{1}$, Yan-Yan Li', Peng Jiao ${ }^{1}$, Hao Wang ${ }^{1}$, Shu-Cun Qin ${ }^{1 *}$ and Shu-Tong Yao ${ }^{1,4^{*}}$

\begin{abstract}
Background: Ethanol extract of propolis (EEP), rich in flavones, has been known for various biological activities including antioxidant, antiinflammatory and antibiotic activities. Our previous studies have shown that EEP protects endothelial cells from oxidized low-density lipoprotein (ox-LDL)-induced apoptosis and inhibits atherosclerotic lesion development. In this present study, we explored the protective effect of EEP on ox-LDL-induced cytotoxicity in macrophages and specifically the endoplasmic reticulum (ER) stress-C/EBP homologous protein (CHOP) pathway-mediated apoptosis.

Methods: EEP was prepared and the total flavonoids content of EEP was determined by the colorimetric method of Chinese Standard (GB/T 20574-2006). The effects of EEP on lipid accumulation, cytotoxicity and apoptosis in RAW264.7 cells induced by ox-LDL or tunicamycin (TM, an ER stress inducer) were assayed using oil red O staining, MTT assay, flow cytometric analysis and so on. Immunofluorescence, Western blot and real time-PCR analysis were then used to further investigate the molecular mechanisms by which EEP protects macrophages from ox-LDL-induced apoptosis. 4-phenylbutyric acid (PBA), an ER stress inhibitor, was used as a positive control.
\end{abstract}

Results: EEP (7.5, 15 and $30 \mathrm{mg} / \mathrm{L})$ not only attenuated ox-LDL-induced lipid accumulation in RAW264.7 macrophages in a dose-dependent manner but also inhibited the decreased cell viability and the increased lactate dehydrogenase (LDH) leakage, caspase-3 activation and apoptosis induced by ox-LDL or tunicamycin (TM, a classical ER stress inducer), which were similar to 4-phenylbutyric acid (PBA, an inhibitor of ER stress) treatment. In addition, like PBA, EEP significantly suppressed the ox-LDL- or TM-induced activation of ER stress signaling pathway including the phosphorylation of double-stranded RNA-activated protein kinase-like ER kinase (PERK) and eukaryotic translation initiation factor 2a (elF2a) as well as upregulation of glucose regulated protein 78 (GRP78) and the pro-apoptotic protein CHOP. Furthermore, EEP significantly suppressed ox-LDL intake by macrophages and the upregulation of CD36 induced by ox-LDL.

Conclusion: These data indicate that EEP may protect macrophages from ox-LDL-induced apoptosis and the mechanism at least partially involves its ability to suppress the CD36-mediated ox-LDL intake and subsequent activation of ER stress-CHOP signalling pathway.

Keywords: Ethanol extract of propolis, Endoplasmic reticulum stress, C/EBP homologous protein, Oxidized low density lipoprotein, Macrophage, Apoptosis

\footnotetext{
*Correspondence: shucunqin@hotmail.com; yst228@126.com

'Key Laboratory of Atherosclerosis in Universities of Shandong, Institute of

Atherosclerosis, Taishan Medical University, Taian 271000, China

Full list of author information is available at the end of the article
} 


\section{Background}

Apoptosis, especially in macrophages-dense atherosclerotic lesions, is unanimously considered as a prominent feature of advanced atherosclerotic plaques, suggesting that macrophage apoptosis is closely related to the atherosclerotic development and subsequent plaque rupture, which is the prominent event that results in the majority of clinical manifestations of acute coronary syndrome such as acute myocardial infarction and sudden coronary death [1]. Macrophage apoptosis at early stages, combined with efficient clearance of apoptotic cells (efferocytosis), helps in maintaining reduced cellularity and slows lesion progression. However in advanced atherosclerotic lesions where efferocytosis is defective, apoptosis of macrophage- derived foam cells contributes to the formation and expansion of lipid core, and gives rise to inflammation and necrosis, which lead to plaque instability [2]. Thus, protecting macrophages from apoptosis is believed as an effective approach to attenuate plaque instability and combat acute vascular events [3].

Propolis, a resinous hive product collected by honeybees from many plant sources, contains predominantly phenolic compounds including flavonoids, phenolic acids and their esters [4-6]. Propolis has a long history of being used in traditional medicine because of its broad spectrum of biological activities incoluding anticancer, antioxidant, antiinflammatory, antibiotic and antifungal properties [7]. Recent studies have shown that ethanol extract of propolis (EEP), rich in flavones, has inhibitory effects on inflammatory responses and inflammation-related transcription factors in macrophages $[8,9]$. In our previous studies, we have found that EEP promotes reverse cholesterol transport and restrians atherosclerotic lesion development [10, 11]. More interestingly, our previous studies have shown that EEP protects endothelial cells from oxidized low density lipoprotein (ox-LDL)-induced injury by inhibiting lectinlike oxidized low density lipoprotein receptor-1 (LOX-1)mediated oxidative stress [12]. However, whether EEP protects macrophages from ox-LDL-induced apoptosis by inhibiting endoplasmic reticulum (ER) stress-C/EBP homologous protein $(\mathrm{CHOP})$ pathway has not yet been determined.

$\mathrm{CHOP}$, belonging to $\mathrm{C} / \mathrm{EBP}$ transcription factor family, is a specific transcription factor in ER stress [13]. ER stress-CHOP pathway-mediated apoptosis in macrophages has been confirmed to promote the instability of atherosclerotic plaques, and the deficiency of $\mathrm{CHOP}$ has been discovered to prevent macrophages from ER stressinduced apoptosis in vitro and in advanced atherosclerotic lesions of mice $[14,15]$. Therefore, a positive correlation among CHOP expression, apoptosis of macrophages and progression of atherosclerotic plaques to the vulnerable stage is undoubted $[2,15,16]$. We have previously reported that both minimally modified LDL (mm-LDL) and ox-LDL can induce ER stress during the formation of macrophage-derived foam cells, and double-stranded RNA-activated protein kinase-like ER kinase (PERK) mediates ox-LDL-induced macrophage apoptosis by upregulating $\mathrm{CHOP}$ expression $[17,18]$. In addition, our earlier study has provided preliminary evidence that quercetin, one of the flavonoids that are ubiquitous in plants and propolis, protects macrophages from ox-LDL-induced apoptosis by inhibiting CHOP expression [19]. Therefore, we hypothesize that EEP may protect macrophages from ox-LDL-induced apoptosis through suppressing ER stress$\mathrm{CHOP}$ signalling pathway. In this present study, we explored the protective effect of EEP on ox-LDL-induced cytotoxicity in RAW264.7 macrophages and specifically the ER stress-CHOP pathway-mediated apoptosis.

\section{Methods \\ Reagents}

Tunicamycin (TM), oil red O, 4-phenylbutyric acid (PBA) and rabbit antibody against $\beta$-actin were purchased from Sigma-Aldrich (St Louis, MO, USA). Dulbecco's modified Eagle medium (DMEM) and fetal bovine serum (FBS) were obtained from Gibco (Rockville, MD, USA). RIPA lysis buffer and DiI-ox-LDL were from Solarbio (Beijing, China) and Xiesheng Biotech (Beijing, China), respectively. Rabbit polyclonal antibodies against glucose regulated proteins 78 (GRP78), CHOP, double-stranded RNA-activated protein kinase-like ER kinase (PERK), eukaryotic translation initiation factor $2 \alpha$ (eIF2 $\alpha$ ) and phospho-eIF $2 \alpha$ (p-eIF $2 \alpha$ ) were purchased from Santa Cruz Biotechnology (Santa Cruz, CA, USA). Rabbit antibodies against phosphoPERK (p-PERK) and anti-CD36 monoclonal antibody $(\mathrm{mAb})$ were purchased from Abcam (Cambridge, MA, USA). SABC-Cy3 immunohistochemistry kits were obtained from Boshide (Wuhan, China). Annexin V-FITC apoptosis detection kits, 3-(4,5-dimethylthiazol- 2-y-1)2,5-diphenyl-2H-tetrazolium bromide (MTT) and lactate dehydrogenase (LDH) assay kits were obtained from BD Biosciences (San Jose, CA, USA), Genview (Houston, TX, USA) and Jiancheng Biotech (Nanjing,China), respectively. Enhanced chemiluminescence (ECL) kits and polyvinylidene fluoride (PVDF) membranes were obtained from Thermo Scientific Pierce (Rockford, IL, USA) and Millipore (Bedford, MA, USA), respectively. Caspase-3 activity assay kit and terminal deoxynucleotidyl transferase-mediated dUTP nick endlabeling (TUNEL) assay kit were from Calbiochem (San Diego, CA, USA) and Roche (Mannheim, Germany), respectively. Real-time PCR reagent kits were purchased from Tiangen Biological Chemistry (Beijing, China). Tissue/cell total cholesterol (TC) assay kits were obtained from Applygen (Beijing, China). 


\section{Preparation of EEP and total flavonoids measurement} Propolis was harvested from Taishan Mountain in Shandong province (China) and EEP was prepared as described in our recent report [12]. Briefly, propolis powder $(100 \mathrm{~g})$ was extracted in $95 \%(\mathrm{v} / \mathrm{v})$ ethanol $(1 \mathrm{~L})$ under the condition of sonication at $40{ }^{\circ} \mathrm{C}$ for three times, and then the supernatant were evaporated in a rotary evaporator under a reduced pressure at $50{ }^{\circ} \mathrm{C}$. Finally, the EEP was dried in the oven and stored at $-20{ }^{\circ} \mathrm{C}$. The total flavonoids content of EEP was $213.46 \pm 2.93 \mathrm{mg}$ rutin equivalent per gram according to the colorimetric method of Chinese Standard (GB/T 20574-2006). Immediately prior to use, EEP sample was dissolved in dimethylsulfoxide (DMSO) and diluted with cell culture medium into appropriate concentrations.

\section{Isolation and oxidation of LDL}

Human LDL was isolated from fresh plasma of healthy donors using sequential ultracentrifugation, and then oxidized with $10 \mu \mathrm{mol} / \mathrm{L} \mathrm{CuSO}_{4}$ for $18 \mathrm{~h}$ at $37^{\circ} \mathrm{C}$ as described in our previous study [17]. The use of human blood and protocol of human LDL isolation were approved by the Ethics Committee of Taishan Medical University, and all blood donors provided informed consent for the use of their blood.

\section{Cell culture}

RAW264.7 macrophages were purchased from the Type Culture Collection of the Chinese Academy of Sciences (Shanghai, China), cultured in DMEM supplemented with $2 \mathrm{mM}$ glutamine, antibiotics $(100 \mathrm{U} / \mathrm{ml}$ penicillin and streptomycin) and $10 \% \mathrm{FBS}$ in a $37{ }^{\circ} \mathrm{C}$ humidified incubator containing $5 \% \mathrm{CO}_{2}$ until subconfluent, and then the medium was replaced with serum-free medium for $12 \mathrm{~h}$ before treatment.

\section{Oil red $\mathrm{O}$ staining}

The intracellular lipid droplets were stained by oil red $\mathrm{O}$ and the lipid droplet content was expressed as the average value of the integrated optical density (IOD) as described previously [17].

\section{Intracellular TC analysis}

The intracellular TC concentration was measured using a tissue/cell TC assay kit according to the manufacturer's instructions and normalized to the level of total cellular protein.

\section{Cell viability and LDH assay}

The viability of the treated cells grown in 96-well plates was evaluated using MTT assay as described previously [17] and cell viability was expressed as the percentage of the control group.
The release of the cytosolic LDH into the medium was used as a generic index of cell injury. After treatment, the media were collected and assayed for LDH activity using a LDH activity assay kit according to the manufacturer's instructions.

\section{Flow cytometry analysis of apoptotic cells}

The Annexin V-FITC/PI double-staining assay was used to quantify apoptosis. Cells of each group were collected, washed with ice-cold PBS twice and centrifuged at $4{ }^{\circ} \mathrm{C}$. $500 \mu \mathrm{L}$ binding buffer was added to resuspend the cells, then $5 \mu \mathrm{L}$ Annexin V-FITC and $5 \mu \mathrm{L}$ PI were added. The cells were incubated for $15 \mathrm{~min}$ in the dark at room temperature, and the apoptosis rates were analyzed on a FACScan flow cytometer using Cell Quest software (Becton Dickinson, San Jose, CA, USA).

\section{TUNEL staining}

DNA strand breaks in the treated macrophages were detected using the TUNEL staining kit according to the manufacturer's instructions. Cells were fixed with $4 \%$ paraformaldehyde for $30 \mathrm{~min}$ at room temperature, rinsed with PBS, and then incubated in $0.1 \%$ triton $\mathrm{X}-100$ permeabilisation solution for $2 \mathrm{~min}$ on ice. The TUNEL reaction mixture was added to the cells and incubated for $1 \mathrm{~h}$ in a dark humidified chamber at $37{ }^{\circ} \mathrm{C}$. After incubation, the cells were washed twice for $5 \mathrm{~min}$ in PBS and stained with 4,6-diamidino-2'-phenylindole dihydrochloride (DAPI). Cells were detected using a fluorescence microscope (Olympus, Tokyo, Japan) and the ratio of TUNEL-positive cells to total cells was calculated.

\section{Measurement of caspase- 3 activity}

Caspase-3 activity was identified with an assay kit according to the manufacturers' instructions. Briefly, following treatment, RAW264.7 cells were harvested, rinsed with PBS and lysed with lysis buffer. The lysate was centrifuged at $12000 \mathrm{~g}$ at $4{ }^{\circ} \mathrm{C}$ for $15 \mathrm{~min}$. $20 \mu \mathrm{l}$ lysate supernatant was added to $70 \mu \mathrm{l}$ reaction buffer and $10 \mu$ caspase- 3 substrate, and then the mixture was incubated in 96-well microtiter plates at $37{ }^{\circ} \mathrm{C}$ for $2 \mathrm{~h}$. Caspase- 3 activity was detected by an Infinite F200 microplate reader (Tecan, Switzerland) at $405 \mathrm{~nm}$ and described as a percentage of the control.

\section{Western blot analysis}

At the end of treatment, the cells were washed and lysed in RIPA buffer. Equal amounts of protein were separated on SDS-PAGE by electrophoresis and then transferred onto a PVDF membrane. After blocking in $10 \%$ nonfat dry milk for $2 \mathrm{~h}$ at room temperature, the membranes were probed with primary antibodies overnight at $4{ }^{\circ} \mathrm{C}$ and then incubated with horseradish peroxidase-conjugated 
secondary antibodies for $1 \mathrm{~h}$ at room temperature. The bound immunoproteins were visualized by ECL reaction, and then the intensities were quantified by ImagePro Plus software (version 6.0, Media Cybernetics, LP, USA) and normalized to $\beta$-actin levels.

\section{Quantitative real time-PCR analysis}

Toal RNA from the treated cells were isolated using Trizol reagent (Invitrogen), and synthesized to cDNA using MuLV Reverse Transcriptase. Real-time PCR were performed on a Rotor-Gene Q real-time PCR cycler (Qiagen, Shanghai, China) using SYBR-green PCR master mix kits as described previously $[17,19]$. The primers were synthesized by Sangon Biotech (Shanghai, China) and the oligonucleotide sequences were as follows: CHOP: 5'CCACCACACCTGAAAGCAGAA-3'(forward primer), 5'GGTGCCCCCAATTTCATCT-3'(reverse primer); GRP78: 5'-ACATGGACCTG TTCCGCTCTA-3' (forward primer), 5'-TGGCTCCTTGCCATTGAAGA-3' (reverse primer); ß-actin: '-CGGGGACCTGACTGACTACC-3' (forward primer), 5'AGGA AGGCTGGAAGAGTGC-3' (reverse primer). The data were analyzed using the Rotor- Gene $Q$ software (version 1.7, Qiagen), and then relative mRNA levels were calculated by the $2^{-\Delta \Delta \mathrm{Ct}}$ method.

\section{Uptake of Dil-ox-LDL}

Cells were pretreated with $\operatorname{EEP}(7.5,15$ and $30 \mathrm{mg} / \mathrm{L})$ or anti-CD36 $\mathrm{mAb}(2 \mathrm{mg} / \mathrm{L})$ for $1 \mathrm{~h}$ and then incubated with Dil-ox-LDL $(50 \mathrm{mg} / \mathrm{L})$ for $6 \mathrm{~h}$. Cells were washed with PBS and lysed in $200 \mu \mathrm{L}$ lysis buffer. Using an Infinite F200 microplate reader (Tecan, Switzerland), the levels of Dil-ox-LDL in cell lysate were quantitated as reported previously [20], and these data were standardized to the protein concentration of each sample.

The uptake of Dil-ox-LDL by RAW264.7 cells was further assayed by fluorescence microscopy. Cells were washed with PBS, fixed with $4 \%$ paraformaldehyde for $20 \mathrm{~min}$, and the cell nuclei was counterstained with DAPI for $20 \mathrm{~min}$. After washing for three times, the cells were observed using a fluorescence microscope (Olympus, Tokyo, Japan) and the mean fluorescence intensity per cell was calculated using Image-Pro Plus software 6.0.

\section{Immunofluorescence assay for CD36 expression}

The treated cells were fixed with $4 \%(\mathrm{w} / \mathrm{v})$ paraformaldehyde, blocked with BSA, and then incubated with CD36 antibody (1:100) overnight at $4{ }^{\circ} \mathrm{C}$. After incubation with secondary FITC-conjugated antibody and DAPI, the cells were washed with PBS, mounted in antifade reagent and then observed using an Olympus BX51 microscopes as described previously [20].

\section{Statistical analysis}

Results are expressed as the mean \pm SEM. Statistical analysis was performed by one-way analysis of variance with Student-Newmann-Keuls multiple comparison tests using the SPSS13.0 software for Windows. P-values less than 0.05 were considered significant.

\section{Results \\ EEP suppresses ox-LDL-induced lipid cumulation in RAW264.7 cells}

Lipid droplets stained by oil red $\mathrm{O}$ were observed significantly in RAW264.7 cells treated with $100 \mathrm{mg} / \mathrm{L}$ of oxLDL for $24 \mathrm{~h}$. However, the ox-LDL-induced cumulation of lipid droplets was reduced by EEP in a dosedependent manner (Fig. 1a and b). Lipid cumulation was further certified by intracellular TC quantitative assay. Compared with the control, intracellular TC content was remarkably increased (3.9-fold) by ox-LDL treatment. However, the effect of ox-LDL was attenuated by $21.3 \%, 28.6 \%$ and $41.3 \%$ upon EEP treatment at 7.5, 15 and $30 \mathrm{mg} / \mathrm{L}$, respectively (Fig. 1c).

\section{EEP attenuates cytotoxicity in RAW264.7 cells induced by ox-LDL or TM}

Cytotoxicity of EEP on RAW264.7 cells was assessed by MTT assay. Except for the concentration of $60 \mathrm{mg} / \mathrm{L}$, stimulation with EEP at $7.5 \mathrm{up}$ to $30 \mathrm{mg} / \mathrm{L}$ for $24 \mathrm{~h}$ had no remarkable effect on cell viability (Fig. $2 \mathrm{a}$ ). Next, we determined the protective effect of EEP on ox-LDL-induced cell death. The treatment with $100 \mathrm{mg} / \mathrm{L}$ ox-LDL for $24 \mathrm{~h}$ reduced cell viability by approximately $47.7 \%$ as measured using MTT assay. However, preincubation with different doses of EEP (7.5, 15 and $30 \mathrm{mg} / \mathrm{L})$ increased the cell viability in a dose-dependent manner compared with cells treated with ox-LDL (Fig. 2b).

$\mathrm{LDH}$, which leaks from cells after plasma membrane injury, was determined to further certify the protective effect of EEP on RAW264.7 cells. As shown in Fig. 2c, LDH release increased dramaticly in the media after cells were incubated with ox-LDL. However, pretreatment with EEP dramaticly reduced the LDH release in a dosedependent manner.

To determine the protective effect of EEP on ER stressmediated cell death, PBA, an ER stress inhibitor, was used as a positive control. As shown in Fig. $2 \mathrm{~b}$ and c, PBA also prevented the decreased cell viability and the LDH release induced by ox-LDL. In addition, TM, an ER stress inducer by inhibiting protein glycosylation, was also used in the study to develop ER stress model. When cells were preincubated with EEP (30 mg/L) before TM $(5 \mathrm{mg} / \mathrm{L})$ treatement for $12 \mathrm{~h}$, the cell viability raised by $32.5 \%$ compared with TM exposure alone, which was similar to PBA pretreatment (Fig. 2d). These data indicated that EEP was able to restrain ER stress-induced cell death. 

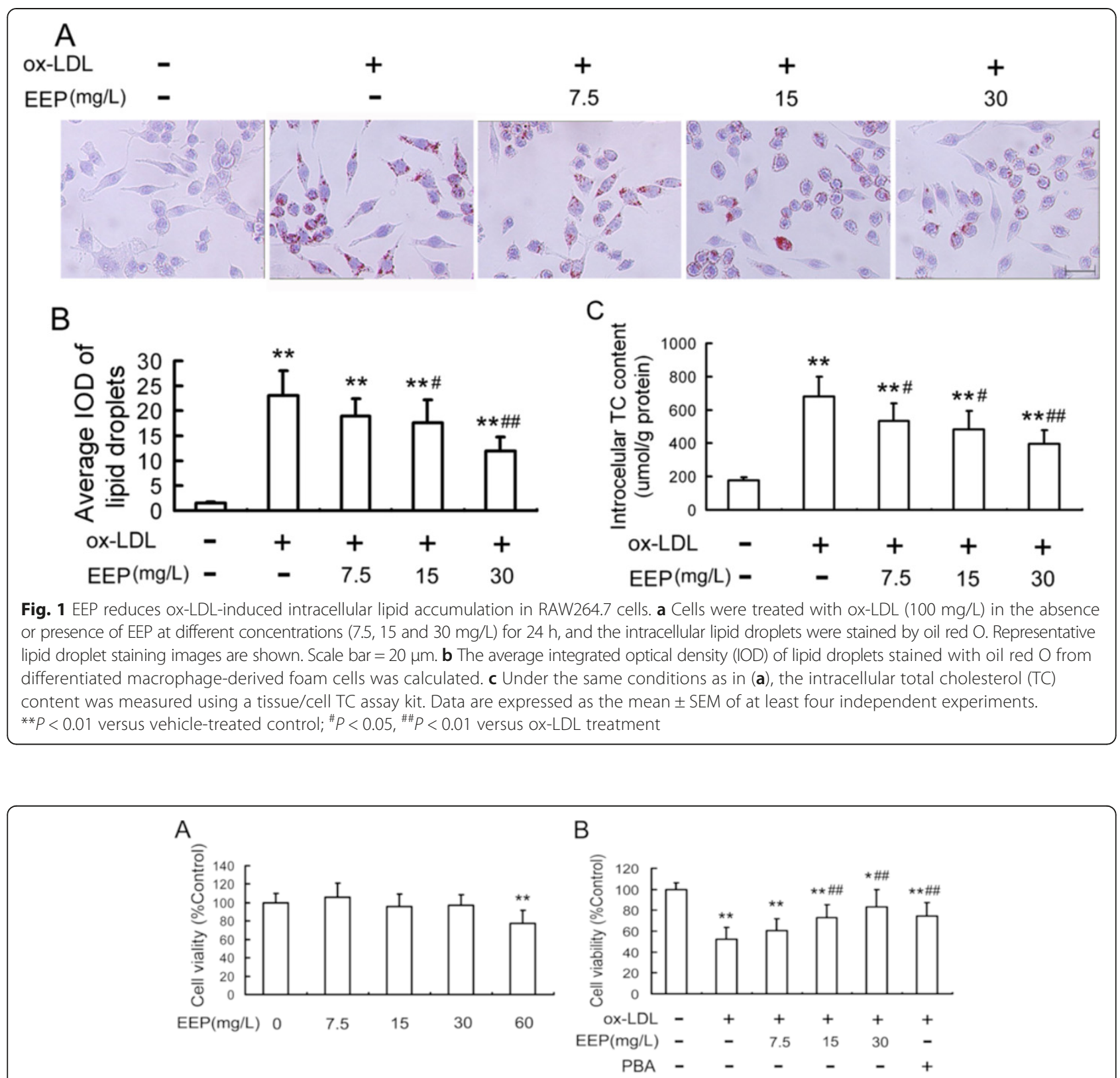

C

$\mathrm{D}$
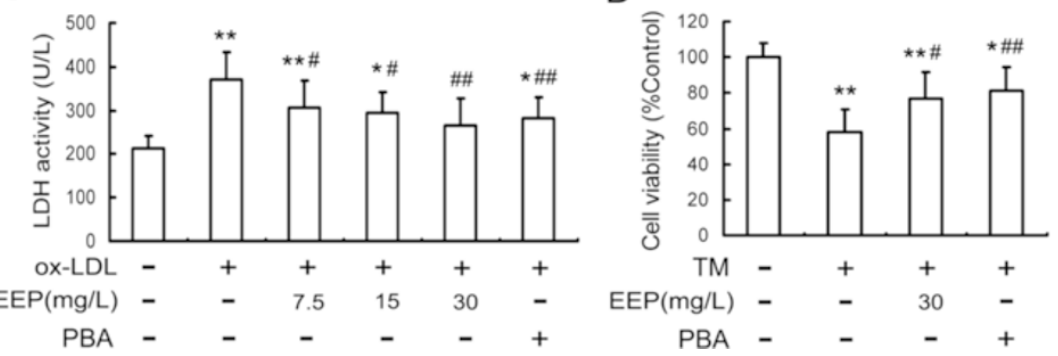

Fig. 2 Effects of EEP on ox-LDL- or TM-induced cytotoxicity in RAW264.7 cells. a Determination of the cytotoxic effects of EEP on RAW264.7 cells. Cells were incubated with the indicated concentrations of EEP for $24 \mathrm{~h}$ and cell viability was measured by the MTT assay and expressed as the percentage of control. $\mathbf{b}$ and $\mathbf{c}$ EEP inhibits ox-LDL-induced cell death and LDH leakage. RAW264.7 cells were pretreated with EEP (7.5, 15 and $30 \mathrm{mg} / \mathrm{L})$ or PBA $(5 \mathrm{mmol} / \mathrm{L})$ for $1 \mathrm{~h}$, and then exposed to ox-LDL (100 mg/L) for $24 \mathrm{~h}$. $\mathbf{d}$ EEP inhibits TM-induced cell death. Cells were pretreated with EEP (30 mg/L) or PBA ( $5 \mathrm{mmol} / \mathrm{L})$ for $1 \mathrm{~h}$, and then incubated with TM $(5 \mathrm{mg} / \mathrm{L})$ for $12 \mathrm{~h}$. Data are expressed as the mean \pm SEM of six independent experiments. ${ }^{*} P<0.05,{ }^{* *} P<0.01$ versus vehicle-treated control; ${ }^{\#} P<0.05,{ }^{\# \#} P<0.01$ versus ox-LDL or TM treatment 


\section{EEP attenuates apoptosis in RAW264.7 cells induced by ox-LDL or TM}

To investigate whether EEP could attenuate ox-LDLinduced apoptosis, the apoptotic rate of the treated cells were analyzed by flow cytometry. RAW264.7 cells were induced apoptosis with ox-LDL (100 mg/L) for $24 \mathrm{~h}$, whereas EEP pretreatment $(7.5,15$ and $30 \mathrm{mg} / \mathrm{L})$ attenuated ox-LDL-induced apoptosis in a dose-dependent manner. Similarly, the inhibitory effect of PBA on oxLDL-induced apoptosis was also observed (Fig. 3a).

TUNEL staining was performed to further confirm the anti-apoptotic effects of EEP. Similar results were observed using cells pretreated with $30 \mathrm{mg} / \mathrm{L}$ EEP or $5 \mathrm{mmol} / \mathrm{L}$ PBA compared with ox-LDL treatment alone (Fig. 3b). Additionally, TUNEL analysis showed that the increase in apoptotic rate induced by TM was attenuated by the addition of EEP (Fig. 3c).

The activity of caspase-3, a marker of apoptosis, was also determined in our study. As illustrated in Fig. 3d and e, the ox-LDL- or TM-induced increase in caspase- 3 activity was remarkably inhibited by EEP in a dose-dependent manner. Moreover, the ox-LDLinduced activation of caspase- 3 was also inhibited by PBA (Fig. 3d).

\section{EEP inhibits ox-LDL-induced ER stress response in RAW264.7 cells}

CHOP is involved in the process of apoptosis associated with ER stress, while PERK is one of important upstream molecules that play an important role in the induction of CHOP. ${ }^{20}$ Since we confirmed that like PBA, EEP could attenuate the ox-LDL- and TM-induced apoptosis in RAW264.7 cells (Fig. 3), we next examined the effect of EEP on ER stress-CHOP pathway. As seen in Fig. 4 a, ox-LDL caused significantly the protein upregulation of p-PERK, p-eIF2 $\alpha$, GRP78 and CHOP in RAW264.7 cells compared with the control cells, which were remarkably inhibited by EEP in a dose-dependent manner. Consistent with the Western blot results, ox-LDLinduced upregulation of CHOP and GRP78 mRNA were also attenuated by EEP (Fig. 4b). Similar results were obtained using the cells pretreated with PBA (Fig. $4 \mathrm{a}$ and b).

\section{EEP inhibits TM-induced ER stress response in RAW264.7 cells}

To further confirm EEP could reduce the apoptosis of RAW264.7 cells by inhibiting ER stress-CHOP pathway, we explored the changes of ER stress markers on TMinduced ER stress model. Similar to the results above, pretreatment with EEP could inhibit TM-induced phosphorylation of PERK and eIF2 $\alpha$ and the upregulation of GRP 78 and CHOP (Fig. 5a and b).

\section{EEP suppresses ox-LDL uptake and CD36 expression in RAW264.7 cells}

Since about $60 \%-70 \%$ of macrophage-derived foam cell formation is caused by CD36-mediated ox-LDL uptake, which is an important factor to lead to ER stress [20, 21], and EEP suppressed ox-LDL-induced lipid cumulation in RAW264.7 cells (Fig. 1), we next detected whether the mechanism underlying the regulatory effect of EEP on ER stress-CHOP pathway could be through inhibition of CD36 expression. Our results demostrated that the uptake of Dil-ox-LDL in RAW264.7 cells was reduced by EEP in a concentration-dependent manner, which was similar to anti-CD36 mAb treatment (Fig. 6a and $b$ ). In addition, Western blot and immunofluorescence results (Fig. $6 \mathrm{c}$ and d) showed that ox-LDLinduced CD36 upregulation was significantly restrained by EEP, suggesting that EEP may inhibit the uptake of ox-LDL via supression of CD36 expression.

\section{Discussion}

Apoptosis of lipid-containing macrophages in the advanced atherosclerotic lesion promotes inflammatory response, lesional necrosis and enlargement of the lipid core, which lead to plaque rupture and acute thrombosis $[1,2]$. Thus, inhibition of macrophage apoptosis may be effective in blocking acute cardiovascular events. In the present study, we first demonstrated that EEP not only inhibited ox-LDL-induced macrophage-derived foam cell formation, injury and apoptosis but also reduced ER stress inducer TM-induced macrophage injury and apoptosis, which were similar to PBA (an ER stress inhibitor) pretreatment. Second, like PBA, EEP suppressed ox-LDL or TM-induced ER stress-CHOP pathway via inhibiting PERK activation. In addition, EEP mitigated ox-LDL uptake by macrophages and inhibited CD36 upregulation induced by ox-LDL. These data indicate that EEP inhibits ox-LDL-induced macrophage apoptosis by suppressing CD36-mediated ox-LDL uptake and subsequent activation of ER stress-CHOP pathway.

In recent years, natural medicine especially the plant flavonoids with cardiovascular protective function gained much attention and were proven to reduce the risk of cardiovascular desease in vitro and in vivo based on different animal models [22]. Icariin (a prenylated flavonol glycoside) attenuates cardiac remodelling through downregulating myocardial apoptosis in rats with congestive heart failure [23]. Wogonin suppresses myocardial apoptosis induced by ischemia/reperfusion in rats [24]. Quercetin (a member of the bioflavonoid family) is confirmed to exhibit antiapoptotic properties in various cell types [25-27]. Acumulating evidence has demonstrated that EEP and its flavones exhibit anti-inflammatory effects in macrophages [8, 9]. In addition, our previous studies have revealed that EEP promotes reverse cholesterol 

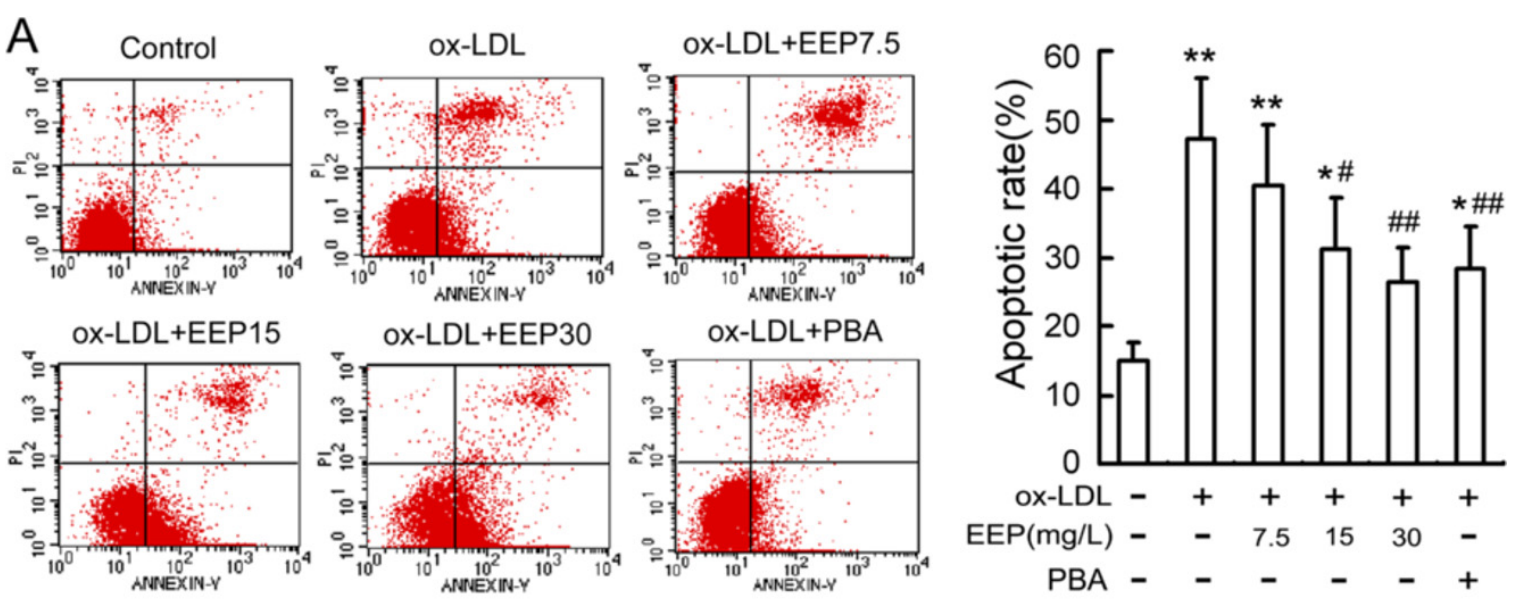

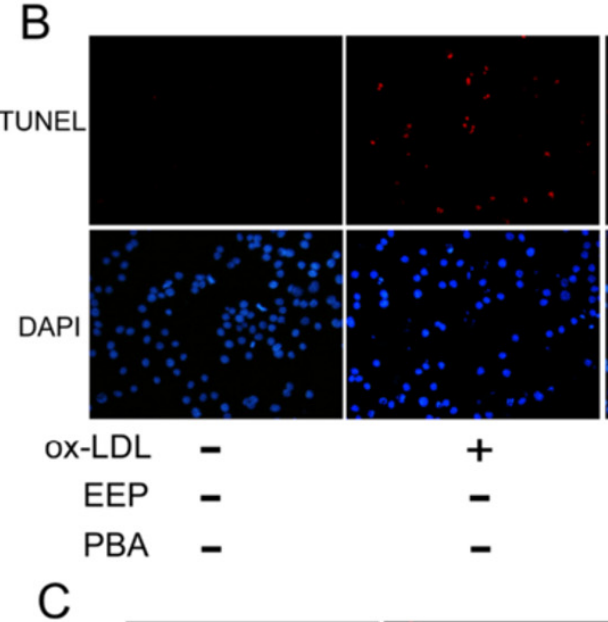

TUNEL
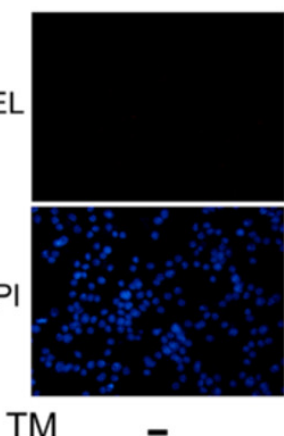

TM EEP

D

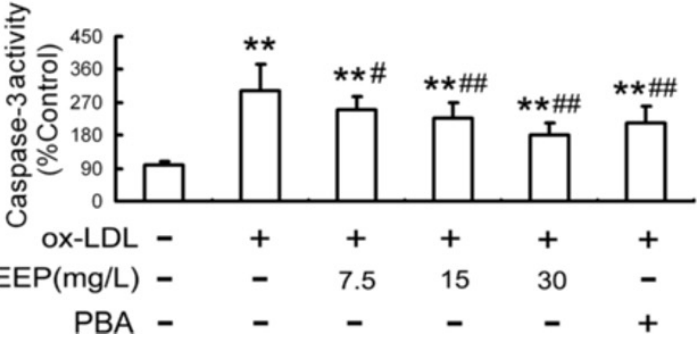

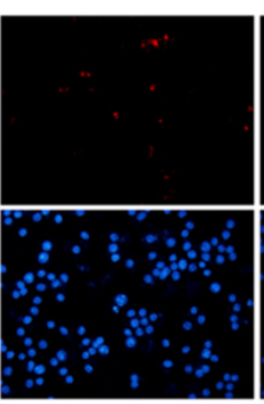

$+$
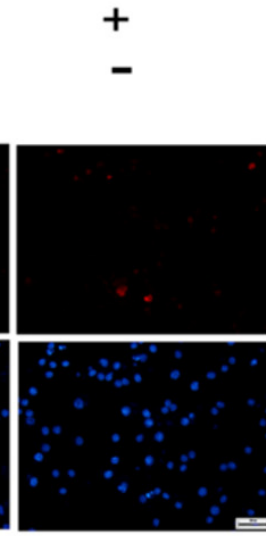

$+$

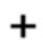

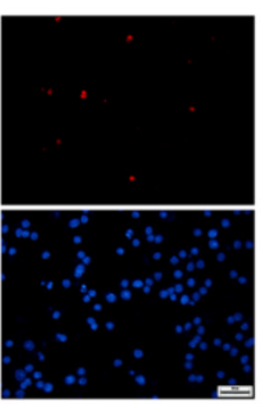

$+$

$-$

$+$

E
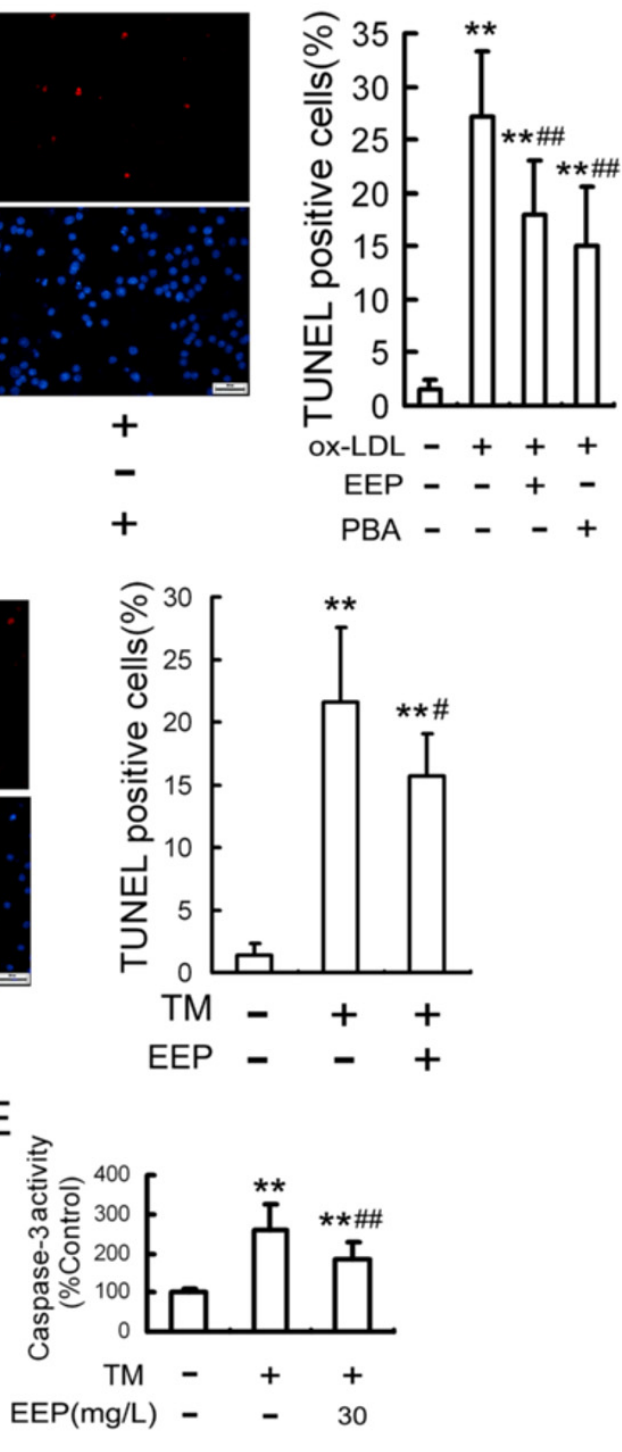

Fig. 3 (See legend on next page.) 
(See figure on previous page.)

Fig. 3 Effect of EEP on ox-LDL- or TM-induced apoptosis in RAW264.7 cells. a After RAW264.7 cells were treated with EEP (7.5, 15 and 30 mg/L) or PBA (5 mmol/L) in the presence of ox-LDL (100 mg/L) treatment for $24 \mathrm{~h}$, cell apoptosis was detected using flow cytometry and the total apoptotic cells (early and late-stage apoptosis) were represented by the right side of the panel (Annexin $V$ staining alone or together with PI). $\mathbf{b}$ and $\mathbf{c}$ Cells were pretreated with EEP (30 mg/L) or PBA ( $5 \mathrm{mmol} / \mathrm{L})$ for $1 \mathrm{~h}$, followed by exposure to ox-LDL (100 mg/L) for $24 \mathrm{~h}$ or TM (5 mg/L) for $12 \mathrm{~h}$, and then cell apoptosis was detected by TUNEL assay. Scale bar $=20 \mu \mathrm{m}$. $\mathbf{d}$ and $\mathbf{e}$ Cells were pretreated with the indicated concentrations of EEP or PBA ( $5 \mathrm{mmol} / \mathrm{L}$ ) in the presence of ox-LDL (100 mg/L) for $24 \mathrm{~h}$ or TM $(5 \mathrm{mg} / \mathrm{L})$ treatment for $12 \mathrm{~h}$, and then caspase-3 activity was determined by colorimetric assay. Data are expressed as the mean \pm SEM of at least four independent experiments. ${ }^{*} P<0.05,{ }^{*} P<0.01$ versus vehicle-treated control; ${ }^{\#} P<0.05$, ${ }^{\# \#} P<0.01$ versus ox-LDL or TM treatment

transport, protects endothelial cells from ox-LDL-induced apoptosis and inhibits atherosclerotic lesion development [10-12]. However, whether EEP could inhibit ox-LDLinduced macrophage apoptosis remains unkown. Our results in the present work showed that EEP remarkably restrained ox-LDL-induced lipid cumulation and macrophage insult as reflected by the increased cell viability and the decreased LDH leakage, caspase-3 activation and apoptosis, suggesting that EEP is able to attenuate oxLDL-induced macrophage-derived foam cell injury.

ER is a critical cell organelle which takes part in cell accommodation and death [28]. Accumulating data have indicated that ER stress-mediated apoptosis is associated with plaque instability in atherosclerosis and its vascular complications [16]. ER stress is well known to activate the unfolded protein response (UPR) as an adaptive response to the disequilibrium in ER homeostasis through transient translational inhibition, upregulation of ER molecular chaperone and activation of ER-associated degradation (ERAD). However, when such correction is not satisfactorily achieved due to severe or prolonged ER stress, the pro-apoptotic signaling pathway will be elicited. One of the important signaling mediators in the ER stress-mediated apoptosis pathway is CHOP $[15,29]$. Numerous studies have demonstrated that CHOP is markedly elevated and contributes to macrophage

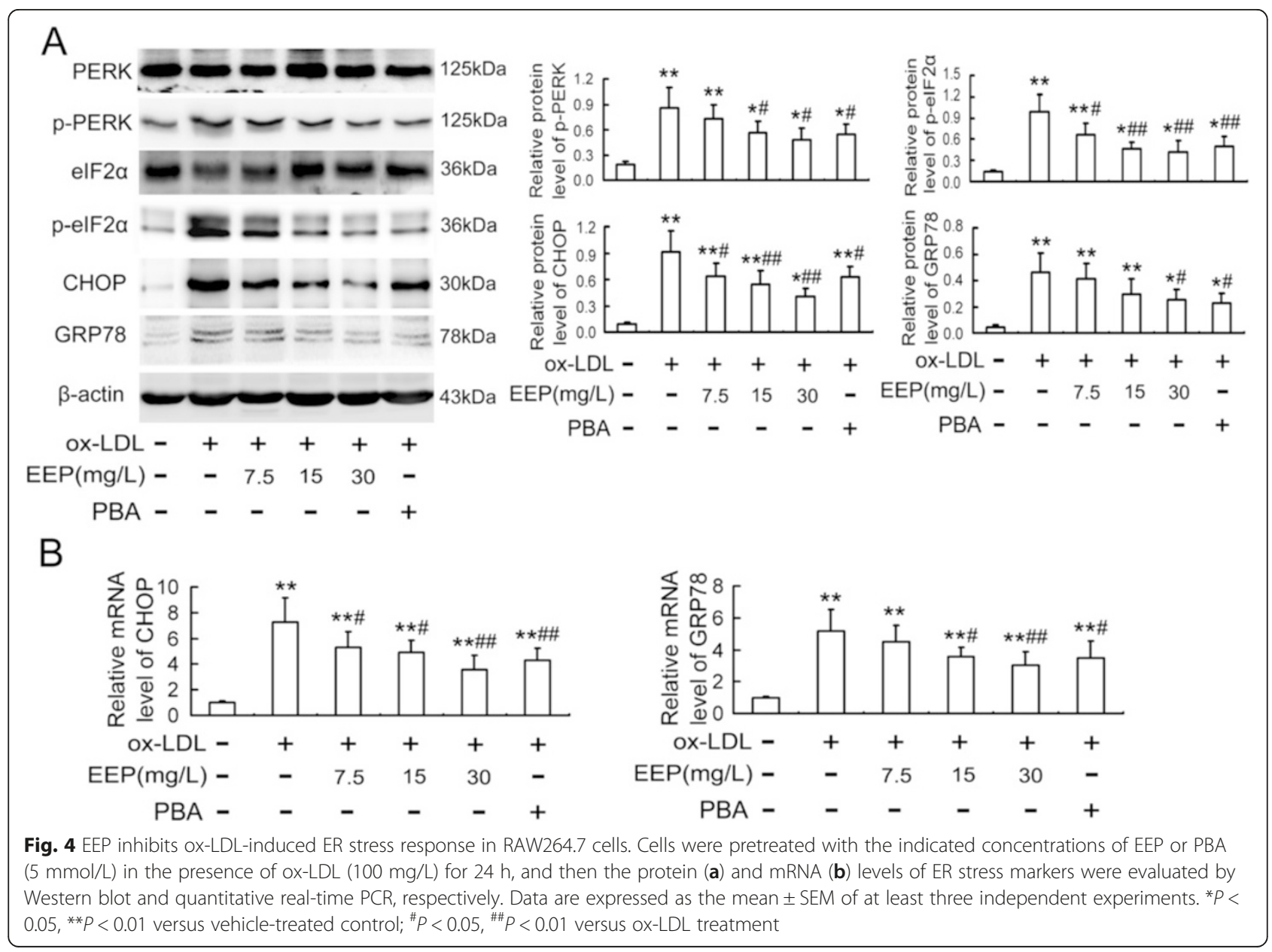



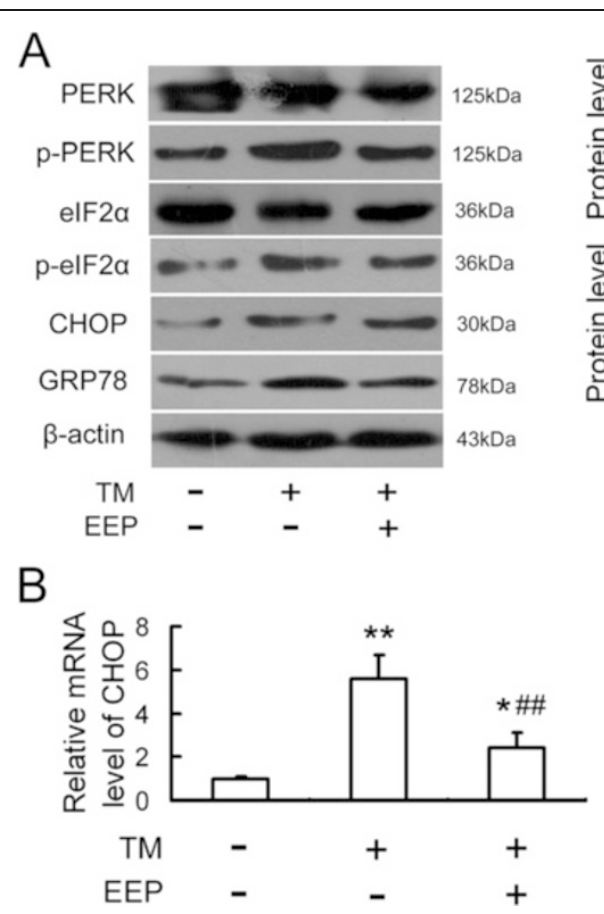
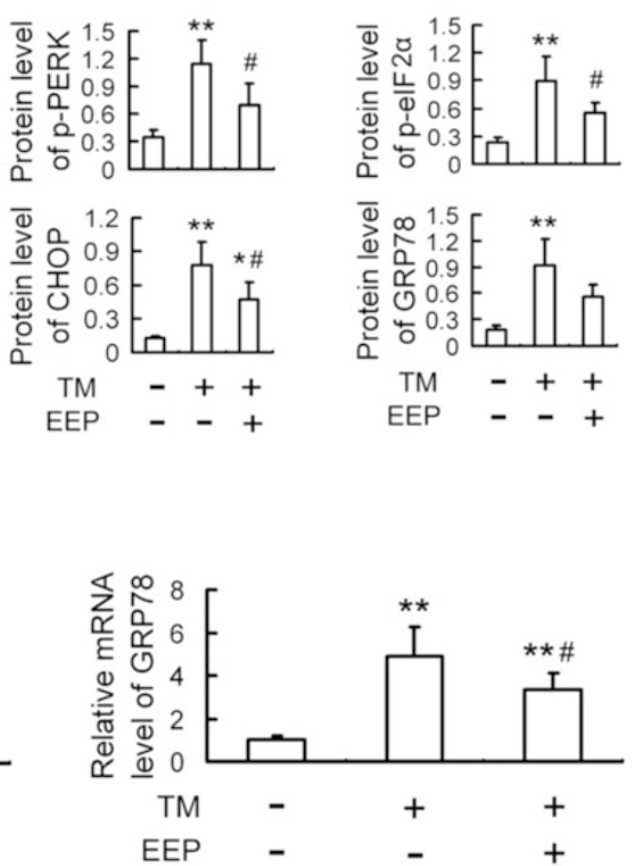

Fig. 5 EEP inhibits TM-induced ER stress response in RAW264.7 cells. Cells were pretreated with EEP (30 mg/L) in the presence of TM (5 mg/L) treatment for $12 \mathrm{~h}$, and then the protein ( $\mathbf{a})$ and mRNA (b) levels of ER stress markers were evaluated by Western blot and quantitative real-time $P C R$, respectively. Data are expressed as the mean \pm SEM of at least three independent experiments. ${ }^{*} P<0.05$, ${ }^{* *} P<0.01$ versus vehicle-treated control; ${ }^{\#} P<0.05,{ }^{\# \#} P<0.01$ versus TM treatment

apoptosis and the instability of atherosclerotic plaques, whereas CHOP deficiency attenuates macrophage apoptosis and atherosclerotic plaque necrosis, suggesting that activation of CHOP is the key signaling step in macrophage apoptosis and plaque instability $[14,15$, 30]. PERK is one of upstream molecules that play a critical role in the induction of CHOP in ER stress. PERK is type I ER transmembrane protein possessing serine/ threonine kinase activity. Activated PERK in response to ER stress induces phosphorylation of eIF $2 \alpha$, thereby reducing the overall protein translational levels, which attenuates the unfolded protein load in ER. In addition, eIF $2 \alpha$ phosphorylation also facilitates the expression of the activating transcription factor 4 (ATF4) that translocates into the nucleus and increases CHOP expression [16]. Our recent work showed that PERK mediated ox-LDL-induced apoptosis in macrophages by upregulating CHOP expression [17]. In the present study, we discovered that EEP downregulated CHOP expression as well as inhibiting the phosphorylation of PERK and eIF $2 \alpha$ induced by ox-LDL in RAW264.7 cells, which was similar to the ER stress inhibitor PBA. TM has been known to facilitate apoptosis by activating ER stress signaling pathways [30]. To further confirm the regulatory effect of EEP on ER stress-CHOP pathways, the TM-induced macrophage apoptosis model was used in the present study. Our data revealed that EEP reduced the TM-induced cytotoxicity and upregulation of CHOP. The phosphorylation of PERK and eIF $2 \alpha$ induced by TM were also dramatically inhibited by EEP. These results indicate that the inhibition of ox-LDLinduced apoptosis by EEP is related to the repression of ER stress-CHOP pathway.

Several observations including our previous studies have demonstrated that the cumulation of intracellular cholesterol is a crucial inducer of ER stress and macrophage apotosis in vivo and in vitro [17, 18, 31, 32]. CD36, a class B scavenger receptor that is expressed on a wide variety of cells especially on monocytes/macrophages, has been identified as the major receptor responsible for ox-LDL uptake into macrophages and cholesterol accumulation [33, 34]. Loss or inhibition of CD36 resultes in significant decreased ability of macrophages to accumulate ox-LDL and cholesteryl, and protectes against atherosclerotic lesion development [35-37]. These data point to a significant role of CD36 in atherosclerosis development and suggest it could be an important target for therapeutic treatment. More interestingly, our recent study has provided that CD36 silencing attenuates ox-LDL-induced ATF6 nuclear translocation and GRP78 upregulation, suggesting that CD36-mediated ox-LDL uptake in macrophages triggers ER stress response [20]. Our present work showed that similar to anti-CD36 antibody, EEP significantly suppressed 


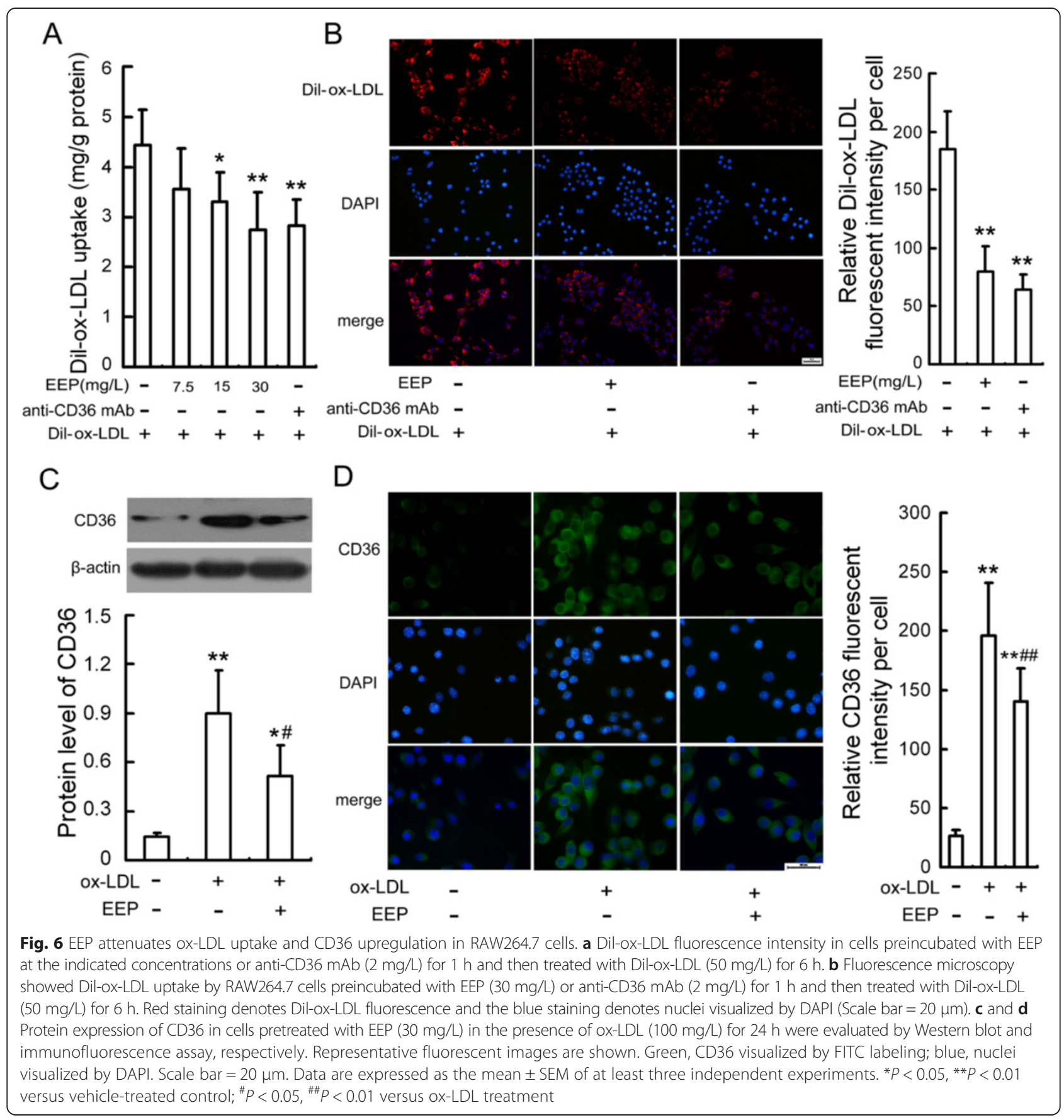

ox-LDL intake as well as the upregulation of CD36 induced by ox-LDL, which may be the mechanism for the inhibitory effect of EEP on the ER stress-CHOP pathwaymediated macrophage apoptosis induced by ox-LDL.

\section{Conclusions}

The present study showed for the first time that EEP protected macrophages from ox-LDL-induced apoptosis through the inhibition of the CD36-mediated ox-LDL intake and subsequent activation of ER stress-CHOP signalling pathway in vitro. The results might explain the diverse physiological activities of EEP and emphasize the pharmacological application of EEP in atherosclerosisrelated diseases.

\section{Abbreviations}

EEP: Ethanol extract of propolis; ox-LDL: oxidized low-density lipoprotein; ER: Endoplasmic reticulum; TC: Total cholesterol; CHOP: C/EBP homologous protein; TM: Tunicamycin; PBA: 4-phenylbutyric acid; PERK: RNA- activated protein kinase-like ER kinase; elF2a: eukaryotic translation initiation factor 2a; GRP78: Glucose regulated protein 78; LOX-1: Lectin-like oxidized low density lipoprotein receptor-1; mm-LDL: minimally modified LDL; 
p-elF2a: phospho- eukaryotic translation initiation factor 2a; p-PERK: phospho-double- stranded RNA- activated protein kinase-like ER kinase; DMSO: Dimethylsulfoxide; IOD: Integrated optical density; LDH: dehydrogenase; MTT: 3-(4,5- dimethylthiazol- 2-y-1)- 2,5- diphenyl-2H-tetrazolium bromide; TUNEL: Terminal deoxynucleotidyl transferase- mediated dUTP nick end-labeling; ATF4: Activating transcription factor 4; DAPI: 4', 6-diamidino-2phenylindole dihydrochloride.

\section{Competing interests}

The authors declare that they have no competing interests.

\section{Authors' contributions}

TH performed the data collection and analysis, and drafted the manuscript. SHW helped to carry out the oil red O staining and MTT assay. ZJJ and ZXW helped to prepare ox-LDL and determine TC, LDH and caspase-3 activity. ZL, GSD and LYY helped to carry out cell culture, immunofluorescence assay, Western blot and quantitative real-time PCR analysis. JP helped to perform flow cytometry analysis. WH helped to prepare EEP. QSC participated in the study design. YST was responsible for the study design, funding, data analysis and manuscript draft. All authors have read and approved the final manuscript.

\section{Authors' information}

${ }^{1}$ Key Laboratory of Atherosclerosis in Universities of Shandong, Institute of Atherosclerosis, Taishan Medical University, Taian 271000, China. ${ }^{2}$ Taishan Hospital of Shandong province, Taian 271000, China. ${ }^{3}$ Affiliated Hospital of Taishan Medical University, Taishan Medical University, Taian 271000, China. ${ }^{4}$ College of Basic Medical Sciences, Taishan Medical University, Taian 271000 China.

\section{Acknowledgements}

This work was supported by National Natural Science Foundation of China (81202949, 81370381), Medical Science and Technology Program of Shandong Province (2013WS0319) and Natural Science Foundation of Shandong Province (ZR2014HL013).

\section{Author details}

'Key Laboratory of Atherosclerosis in Universities of Shandong, Institute of Atherosclerosis, Taishan Medical University, Taian 271000, China. ${ }^{2} T a i s h a n$ Hospital of Shandong province, Taian 271000, China. ${ }^{3}$ Affiliated Hospital of Taishan Medical University, Taishan Medical University, Taian 271000, China. ${ }^{4}$ College of Basic Medical Sciences, Taishan Medical University, Taian 271000 China.

\section{Received: 1 February 2015 Accepted: 2 July 2015}

\section{Published online: 14 July 2015}

\section{References}

1. Thorp E, Tabas I. Mechanisms and consequences of efferocytosis in advanced atherosclerosis. J Leukoc Biol. 2009;86(5):1089-95.

2. Tabas I. Macrophage apoptosis in atherosclerosis: consequences on plaque progression and the role of endoplasmic reticulum stress. Antioxid Redox Signal. 2009;11(9):2333-9.

3. Tiwari RL, Singh V, Barthwal MK. Macrophages: an elusive yet emerging therapeutic target of atherosclerosis. Med Res Rev. 2008;28(4):483-544.

4. Farooqui T, Farooqui AA. Beneficial effects of propolis on human health and neurological diseases. Front Biosci (Elite Ed). 2012;4:779-93.

5. Medic'-Saric' M, Rastija V, Bojic' M, Males Z. From functional food to medicinal product: systematic approach in analysis of polyphenolics from propolis and wine. Nutr J. 2009:8:33-50.

6. Teixeira EW, Message D, Negri G, Salatino A, Stringheta PC. Seasonal varia -tion, chemical composition and antioxid an tactivity of Brazilian propolis samples. Evid Based Complement Alternat Med. 2010;7(3):307-15.

7. Bankova V, Popova M, Trusheva B. Propolis volatile compounds: chemical diversity and biological activity: a review. Chem Cent J. 2014:8:28-35.

8. Blonska M, Bronikowska J, Pietsz G, Czuba ZP, Scheller S, Krol W. Effects of ethanol extract of propolis (EEP) and its flavones on inducible gene expression in J774A.1 macrophages. J Ethnopharmacol. 2004;91(1):25-30.

9. Wang K, Ping S, Huang S, Hu L, Xuan H, Zhang C, et al. Molecular mechanisms underlying the in vitro anti-inflammatory effects of a flavonoid-rich ethanol extract from Chinese propolis (poplar type). Evid Based Complement Alternat Med. 2013;2013:127672-82.
10. Fang $Y$, Sang $H$, Yuan N, Sun H, Yao S, Wang J, et al. Ethanolic extract of propolis inhibits atherosclerosis in ApoE-knockout mice. Lipids Health Dis. 2013;12:123-8

11. Yu Y, Si Y, Song G, Luo T, Wang J, Qin S. Ethanolic extract of propolis promotes reverse cholesterol transport and the expression of ATP-binding cassette transporter A1 and G1 in mice. Lipids. 2011;46(9):805-11.

12. Fang $Y, L i$ J, Ding $M, X u X$, Zhang J, Jiao $P$, et al. Ethanol extract of propolis protects endothelial cells from oxidized low density lipoprotein-induced injury by inhibiting lectin-like oxidized low density lipoprotein receptor-1mediated oxidative stress. Exp Biol Med. 2014;239(12):1678-87.

13. Gargalovic PS. Atherogenesis on the chopping block. Cell Metab. 2009;9(5):399-401.

14. Thorp E, Li G, Seimon TA, Kuriakose G, Ron D, Tabas I. Reduced apoptosis and plaque necrosis in advanced atherosclerotic lesions of Apoe-/- and Ldlr-/- mice lacking CHOP. Cell Metab. 2009:9(5):474-81.

15. Tsukano H, Gotoh T, Endo M, Miyata K, Tazume H, Kadomatsu T, et al. The endoplasmic reticulum stress-C/EBP homologous protein pathway-mediated apoptosis in macrophages contributes to the instability of atherosclerotic plaques. Arterioscler Thromb Vasc Biol. 2010;30(10):1925-32.

16. Zhou AX, Tabas I. The UPR in atherosclerosis. Semin Immunopathol. 2013;35(3):321-32

17. Yao $S$, Zong $C$, Zhang $Y$, Sang $H$, Yang $M$, Jiao $P$, et al. Activating transcription factor 6 mediates oxidized LDL-induced cholesterol accumulation and apoptosis in macrophages by up-regulating CHOP expression. J Atheroscler Thromb. 2013;20(1):94-107.

18. Yao S, Yang N, Song G, Sang H, Tian H, Miao C, et al. Minimally modified low-density lipoprotein induces macrophage endoplasmic reticulum stress via toll-like receptor 4. Biochim Biophys Acta. 2012;1821(7):954-63.

19. Yao S, Sang H, Song G, Yang N, Liu Q, Zhang Y, et al. Quercetin protects macrophages from oxidized low-density lipoprotein- induced apoptosis by inhibiting the endoplasmic reticulum stress-C/EBP homologous protein pathway. Exp Biol Med (Maywood). 2012;237(7):822-31.

20. Yao S, Miao C, Tian H, Sang H, Yang N, Jiao P, et al. Endoplasmic Reticulum Stress Promotes Macrophage-derived Foam Cell Formation by Up-regulating Cluster of Differentiation 36 (CD36) Expression. J Biol Chem. 2014:289(7):4032-42.

21. Kunjathoor W, Febbraio M, Podrez EA, Moore KJ, Andersson L, Koehn S, et al. Scavenger receptors class $\mathrm{A}-\mathrm{I} / \mathrm{II}$ and CD36 are the principal receptors responsible for the uptake of modified low density lipoprotein leading to lipid loading in macrophages. J Biol Chem. 2002;277(51):49982-8.

22. Salvamani S, Gunasekaran B, Shaharuddin NA, Ahmad SA, Shukor MY. Antiartherosclerotic effects of plant flavonoids. Biomed Res Int. 2014:2014:480258-68.

23. Song YH, Cai H, Gu N, Qian CF, Cao SP, Zhao ZM. Icariin attenuates cardiac remodelling through down-regulating myocardial apoptosis and matrix metalloproteinase activity in rats with congestive heart failure. J Pharm Pharmacol. 2011;63(4):541-9.

24. Lee YM, Cheng PY, Chen SY, Chung MT, Sheu JR. Wogonin suppresses arrhythmias, inflammatory responses, and apoptosis induced by myocardial ischemia/reperfusion in rats. J Cardiovasc Pharmacol. 2011;58(2):133-42.

25. Zhang ZJ, Cheang LC, Wang MW, Lee SM. Quercetin exerts a neuro- protective effect through inhibition of the iNOS/NO system and pro-inflammation gene expression in PC12 cells and in zebrafish. Int J Mol Med. 2011;27(2):195-203.

26. Mojzisova G, Sarissky M, Mirossay L, Martinka P, Mojzis J. Effect of flavonoids on daunorubicin-induced toxicity in $\mathrm{H} 9 \mathrm{c} 2$ cardiomyoblasts. Phytother Res. 2009;23(1):136-9.

27. Choi JS, Kang SW, Li J, Kim JL, Bae JY, Kim DS, et al. Blockade of oxidized LDL-triggered endothelial apoptosis by quercetin and rutin through differential signaling pathways involving JAK2. J Agric Food Chem. 2009:57(5):2079-86.

28. Ferri KF, Kroemer G. Organelle-specific initiation of cell death pathways. Nat Cell Biol. 2001:3(11):E255-63.

29. Miyazaki Y, Kaikita K, Endo M, Horio E, Miura M, Tsujita K, et al. C/EBP homologous protein deficiency attenuates myocardial reperfusion injury by inhibiting myocardial apoptosis and inflammation. Arterioscler Thromb Vasc Biol. 2011;31(5):1124-32.

30. Feng B, Yao PM, Li Y, Devlin CM, Zhang D, Harding HP, et al. The endoplasmic reticulum is the site of cholesterol-induced cytotoxicity in macrophages. Nat Cell Biol. 2003;5(9):781-92.

31. Scull CM, Tabas I. Mechanisms of ER stress-induced apoptosis in atherosclerosis. Arterioscler Thromb Vasc Biol. 2011:31(12):2792-7. 
32. Li F, Guo Y, Sun S, Jiang X, Tang B, Wang Q, et al. Free cholesterol-induced macrophage apoptosis is mediated by inositol- requiring enzyme 1 alpha-regulated activation of Jun N-terminal kinase. Acta Biochimica et Biophysica Sinica (Shanghai). 2008;40(3):226-34.

33. Collot-Teixeira S, Martin J, McDermott-Roe C, Poston R, McGregor JL. CD36 and macrophages in atherosclerosis. Cardiovasc Res. 2007;75(3):468-77.

34. Yu XH, Fu YC, Zhang DW, Yin K, Tang CK. Foam cells in atherosclerosis. Clin Chim Acta. 2013;424:245-52.

35. Chávez-Sánchez L, Garza-Reyes MG, Espinosa-Luna JE, Chávez-Rueda K, Legorreta-Haquet MV, Blanco-Favela F. The role of TLR2, TLR4 and CD36 in macrophage activation and foam cell formation in response to oxLDL in humans. Hum Immunol. 2014;75(4):322-9.

36. Park YM. CD36, a scavenger receptor implicated in atherosclerosis. Exp Mol Med. 2014;46:e99-105.

37. Syväranta S, Alanne-Kinnunen M, Oörni K, Oksjoki R, Kupari M, Kovanen PT, et al. Potential pathological roles for oxidized low-density lipoprotein and scavenger receptors SR-Al, CD36, and LOX-1 in aortic valve stenosis. Atherosclerosis. 2014;235(2):398-407.

\section{Submit your next manuscript to BioMed Central and take full advantage of:}

- Convenient online submission

- Thorough peer review

- No space constraints or color figure charges

- Immediate publication on acceptance

- Inclusion in PubMed, CAS, Scopus and Google Scholar

- Research which is freely available for redistribution 